\title{
Blocking Effect of Chaga Mushroom (Inonotus oliquus) Extract for Immune Checkpoint CTLA-4/CD80 Interaction
}

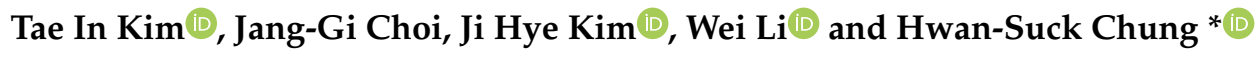 \\ Korean Medicine (KM)-Application Center, Korea Institute of Oriental Medicine (KIOM), Daegu 41062, Korea; \\ tikim@kiom.re.kr (T.I.K.); jang-gichoi@kiom.re.kr (J.-G.C.); jkim2903@kiom.re.kr (J.H.K.); \\ liwei1986@kiom.re.kr (W.L.) \\ * Correspondence: hschung@kiom.re.kr; Tel.: +82-53-940-3875; Fax: +82-53-940-3899
}

Received: 29 July 2020; Accepted: 18 August 2020; Published: 20 August 2020

\begin{abstract}
Inonotus obliquus, also known as the Chaga mushroom, has been used as a traditional medicine to treat many different diseases in Asia. Ethanol and water extraction were performed to examine the blocking effect of the Chaga mushroom on the CTLA-4/CD80 interaction. The inhibitory activities of the Chaga mushroom/70\% EtOH extract (CME) and the Chaga mushroom/water extract (CMW) were confirmed using several cell-based assays. To identify the contents of major compounds CME and CMW, we performed HPLC analysis. The content of lanosterol (1) in CME was $0.41 \%$. Our findings provide experimental evidence that the Chaga mushroom can develop a small-molecule inhibitor that blocks the CTLA-4/CD80 interaction.
\end{abstract}

Keywords: immune checkpoints; CTLA-4; CD80; Inonotus obliquus

\section{Introduction}

Inonotus obliquus, commonly known as the Chaga mushroom, belongs to the family Hymenochaetaceae, and is a parasite mainly found on the birch tree (betula platyphlla var. japonica). It is widely distributed in Russia, China, and Alaska, and has diverse bioactivities, such as anti-inflammatory effects [1], anti-nociceptive effects [2], and inhibition of cell cycle progression [3]. Several constituents, including triterpenoids [4,5], phenolic acids [6], and polysaccharides [7], have previously been reported as contributing to these bioactivities. The Chaga mushroom has been used for a long time as a medicinal mushroom in Asia, Russia, and North America to treat various human diseases, such as gastrointestinal disorders, cardiovascular diseases, and diabetes [8]. Although research on the Chaga mushroom is ongoing, its potential to block immune checkpoints, especially CTLA-4/CD80, is not well understood. We previously reported compounds derived from the medicinal plant that inhibit the immune checkpoint interactions PD-1/PD-L1 and CTLA-4/CD80 [9]. Here, we investigate the blocking effect of Chaga mushroom extract on the CTLA-4/CD80 interaction, by using in vitro assay and HPLC analysis.

When a $\mathrm{T}$ cell is exposed to a pathogen, an immune checkpoint-regulated immune response and an immune reaction by immune checkpoints are both essential for establishing immunological equilibrium. In lymph nodes, the interaction between CD80 on antigen-presenting cells (APCs) and CD28 on the naive $\mathrm{T}$ cell leads to $\mathrm{T}$ cell activation. Activated $\mathrm{T}$ cells are the key lymphocytes of the immune response, and cytotoxic T cells kill cancer cells. Among several immune checkpoints, CTLA-4 is expressed on naive T cells and interacts with CD80 molecules on cancer cells. The binding of CTLA- 4 with CD80 then produces an inhibitory signal during the primary phase of $\mathrm{T}$ cell activation, resulting in the inhibition of $\mathrm{T}$ cell activation and a decreased immune response [10]. Cancer cells use this reaction to avoid the immune system and, consequently, survive in the body. Blocking the CTLA-4/CD80 interaction is one of the key targets for cancer immunotherapy, and has become a successful therapeutic strategy. The first 
immune checkpoint inhibitor to be developed, CTLA-4 mAb (ipilimumab), was approved by the US Food and Drug Administration (FDA). However, patients treated with Ipilimumab suffered side effects, such as systemic, dermatologic, and respiratory issues. Additionally, challenges exist with monoclonal antibodies (mAbs) that are yet to be overcome, such as their high cost, long half-life, large molecular weight, and the possibility of immune-related adverse events [11,12]. Thus, the development of small-molecule inhibitors that interfere with the CTLA-4/CD80 interaction offers a solution to the disadvantages of drug discovery. In this study, we investigated an immune checkpoint inhibitor that blocks the CTLA-4/CD80 interaction, using a cell-based assay and HPLC analysis.

\section{Materials and Methods}

\subsection{Plant Materials}

Dried Inonotus obliquus was purchased from the Bomyeong Herbal Market, Seoul in 2019. Its identity was confirmed (by Dr. Wei Li). A voucher specimen (KIOM-26) was deposited at the Herbarium of Korean Medicine-Application Center, Korea Institute of Oriental Medicine, Republic of Korea.

\subsection{Extraction}

The dried Inonotus obliquus (50.0 g) was chopped and extracted with $70 \% \mathrm{EtOH}$ using reflux, and the extract was filtered; then, the filtrate was evaporated to dryness $(6.81 \mathrm{~g})$. The dried Inonotus obliquus $(50.0 \mathrm{~g})$ was extracted by water, and the water solution was frozen and dried $(40.0 \mathrm{~g})$.

\subsection{Instruments}

The HPLC system used was a Thermo Scientific Dionex UltiMate 3000 system equipped with a binary pump, auto-sampler, diode array UV/VIS detector DAD, and column oven (Dionex Corp., Sunnyvale, CA, USA). The Corona CAD detector (Thermo Fisher, Counteaboeuf, France) was linked to the HPLC system. Highly pure nitrogen for the CAD detector was produced by a nitrogen generator at a nitrogen pressure of 63.8. Chromeleon 7 software (Thermo Fisher, Counteaboeuf, France) was used for data acquisition and processing.

\subsection{Preparation of Standard and Sample Solutions}

The $70 \% \mathrm{EtOH}$ and Chaga mushroom water were extracted using an ultrasonicator (JAC Ultrasonic JAC-3010). Each sample was accurately weighed $(300 \mathrm{mg})$ and dissolved in $10 \mathrm{~mL}$ of methanol for $30 \mathrm{~min}$. After extraction, each solution was filtered through a $0.2 \mu \mathrm{m}$ membrane. A $10 \mu \mathrm{L}$ aliquot of the filtrate was analyzed using HPLC. A standard solution of lanosterol, betulin, and betulinic acid was prepared at $1.0 \mathrm{mg} / \mathrm{mL}$ (1000 ppm) with acetonitrile, and stored at $4{ }^{\circ} \mathrm{C}$ until use. A standard curve of the solution, diluted using methanol at each concentration, was created.

\subsection{Chemicals and Antibodies}

The CTLA-4/CD80 Inhibitor Screening Assay Kit (\#72009) was purchased from BPS Bioscience (San Diego, CA, USA). Anti-CTLA-4 antibody (\#A2001) was purchased from Selleck (Houston, TX, USA). Lanosterol, betulin, and betulinic acid were purchased from Sigma-Aldrich (St. Louis, MO, USA). HPLC-grade acetonitrile was purchased from JT Baker (Philiosburg, NJ, USA). ACS reagent-grade formic acid was obtained from Sigma-Aldrich (St. Louis, MO, USA, purity of $\geq 99.0 \%$ ). Water was prepared using a Puris-Evo RO water system (Mirae ST Co., Ltd., Anyang-si, South Korea). All solutions prepared for HPLC were filtered through $0.2 \mu \mathrm{m}$ membrane filters before use.

\subsection{Competitive ELISA}

Competitive ELISA was performed using a CTLA-4/CD80 Inhibitor Screening Assay Kit, according to the supplier's instructions. Briefly, Fc-tagged human CTLA-4 (BPS Bioscience, \#71149) was coated 
onto a 96-well plate overnight. After washing, vehicle or test samples were diluted in solvent, and then added to each coated well. As a positive control, anti-CTLA- 4 neutralizing antibodies were used. After $1 \mathrm{~h}$ of reaction with the compound, biotinylated hCD80 (BPS Bioscience, \#71114) was added to each well, and the plates were incubated for $2 \mathrm{~h}$ at room temperature. After three washes with PBS-T, diluted streptavidin horseradish peroxidase (HRP) was added to each well, and the plates were reacted for $1 \mathrm{~h}$ while shaking at a low speed. After the reaction, HRP substrates A and B were added. Relative chemiluminescence was measured on a SpectraMax L luminometer (Molecular Devices, San Jose, CA, USA) and expressed as the relative binding activity. The results were normalized to the relative percentage of the vehicle control group. The half-maximal inhibitory concentration $\left(\mathrm{IC}_{50}\right)$ was calculated using the Prism "log[inhibitor] vs. normalized response" equation. All results are presented as the means of three independent biological replicates, with ${ }^{*} p<0.05,{ }^{* *} p<0.01$, and ${ }^{* * *} p<0.001$, and compared with the vehicle control group $[9,13,14]$.

\subsection{CTLA-4 Blockade Bioassay}

The bioluminescent cell-based assay was performed using a CTLA-4 blockade bioassay (Promega, Madison, WI, USA, \#JA4001), according to the supplier's instructions. To identify CMW and CME for inhibitory activity, $5 \times 10^{3}$ CD80/aAPC CHO-K1 cells were pre-incubated with F-12 (10\% FBS) into a 96-well plate. After $24 \mathrm{~h}, \mathrm{CTLA}-4$ antibodies or CMW and CME were added by co-culture to the $1 \times 10^{4}$ CTLA-4 Jurkat T cells. Following mixing with Bio-Glo ${ }^{\mathrm{TM}}$ reagent (Promega, \#G7940), the luminescence was measured using a GloMax ${ }^{\circledR}$ explorer multimode microplate reader (Promega). The fold induction of nuclear factor of the activated T cell (NFAT) luciferase reporter expression was calculated from the sample treated wells/untreated control wells.

\subsection{HPLC Analytical Methods}

We performed HPLC profiling of the Chaga mushroom/70\% EtOH extract (CME) and the Chaga mushroom/water extract (CMW) for the mushroom's three major compounds: lanosterol, betulin, and betulinic acid. HPLC analysis was performed on a Phenomenex Luna C18 column $(250 \mathrm{~mm} \times 4.6 \mathrm{~mm}, 5 \mu \mathrm{m})$ coupled to a $\mathrm{C} 18$ guard cartridge $(4.0 \mathrm{~mm} \times 3.0 \mathrm{~mm})$. The mobile phase was applied at a flow rate of $1 \mathrm{~mL} / \mathrm{min}$, with a gradient of $0.1 \%$ formic acid in water (eluent A) and acetonitrile (eluent B), which included the gradient elution as follows: $0-20 \mathrm{~min}, 90-100 \% \mathrm{~B}$; $20-50 \mathrm{~min}, 100 \% \mathrm{~B} ; 50-60 \mathrm{~min}, 90 \% \mathrm{~B}$. The chromatogram was monitored using a CAD detector. The column temperature was set to $30^{\circ} \mathrm{C}$, the auto-sampler was set to $20^{\circ} \mathrm{C}$, and the injection volume was $10 \mu \mathrm{L}$. Calibration curves, assessed by standard solution and the limits of detection (LOD) and quantification (LOQ) under the chromatographic conditions, were determined by injecting a series of standard solutions until the signal-to-noise $(\mathrm{S} / \mathrm{N})$ ratio for each compound was 3 for LOD and 10 for LOQ. Each sample was analyzed as three preparations from three parallel determinations. Data were collected and analyzed using Chromeleon 7 software (Thermo Fisher, Counteaboeuf, France).

\subsection{Cell Viability in CTLA-4 Jurkat T Cells and CD80/aAPC CHO-K1 Cells}

The cell viabilities of CME and CMW were measured using the CCK-8 assay (\#CK04, Dojindo Molecular Technologies, Inc., Rockville, MD, USA), according to the manufacturer's instructions. Briefly, cells were placed in 96-well plates at a density of $1 \times 10^{4}$ cells/well and were cultured for $24 \mathrm{~h}$. After incubation, CME $(0-200 \mu \mathrm{g} / \mathrm{mL})$ and CMW $(0-200 \mu \mathrm{g} / \mathrm{mL})$ were treated and incubated for $24 \mathrm{~h}$. At this point, the cells were treated with $10 \mu \mathrm{L}$ of CCK solution, and the mixture was kept for $2 \mathrm{~h}$ at $37^{\circ} \mathrm{C}$. A microplate reader (Molecular Devices i3, San Jose, CA, USA) was used to measure the absorbance at $450 \mathrm{~nm}$. 


\section{Results}

\subsection{Chaga Mushroom Extract-Blocked CTLA-4/CD80 Interaction}

To test whether the Chaga mushroom extract blocked the CTLA-4/CD80 interaction, we performed a binding inhibition assay using a competitive CTLA-4/CD80 enzyme-linked immunosorbent assay (ELISA) kit. When well-coated CTLA-4 binds to the biotinylated CD80, it produces chemiluminescence, which can then be measured using a chemiluminescence reader. The presence of our sample can block the CTLA-4/CD80 interaction so that it does not produce chemiluminescence. We observed that anti-CTLA-4 neutralizing antibodies completely inhibited the CTLA-4/CD80 binding ( $\left.\mathrm{IC}_{50} 0.29 \mu \mathrm{g} / \mathrm{mL}\right)$. Chaga mushroom/70\% ethanol extract (CME) and Chaga mushroom/water extract (CMW) showed a concentration-dependent inhibition of CTLA-4/CD80 association with $\mathrm{IC}_{50}$ values of $24.05 \mu \mathrm{g} / \mathrm{mL}$ and $45.24 \mu \mathrm{g} / \mathrm{mL}$, respectively. CMW and CME at $50 \mu \mathrm{g} / \mathrm{mL}$ showed inhibitory effects similar to those of the CTLA-4-blocking antibodies at $0.1 \mu \mathrm{g} / \mathrm{mL}$ and $1.0 \mu \mathrm{g} / \mathrm{mL}$, respectively (Figure 1).

A

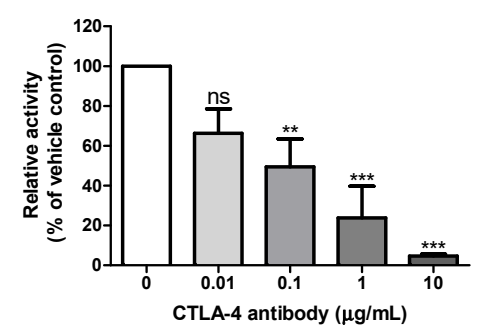

B

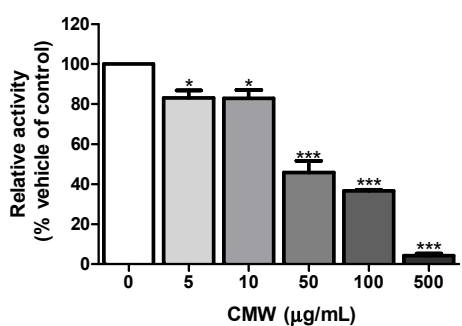

0

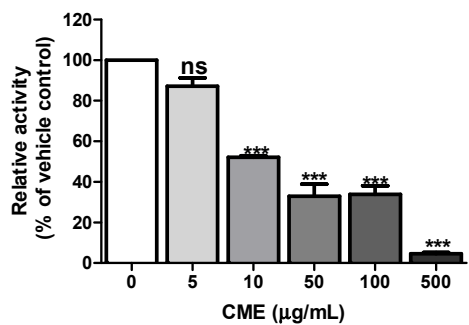

Figure 1. Blocking effect of the CTLA-4/CD80 interaction of CMW and CME by competitive enzyme-linked immunosorbent assay (ELISA). Effect of anti-CTLA-4 neutralizing antibody (A); water extract (B); and 70\% ethanol extract (C) of Chaga mushroom on the CTLA-4/CD80 binding activity. The relative binding activity was normalized to the relative percentage of the vehicle control group. The half-maximal inhibitory concentration $\left(\mathrm{IC}_{50}\right)$ was calculated using the Prism "log[inhibitor] vs. the normalized response" equation. All results are presented as the mean of three independent biological replicates, with ${ }^{*} p<0.05,{ }^{* *} p<0.01,{ }^{* * *} p<0.001$, compared with the vehicle control group.

\subsection{NFAT Reporter Activity of the Chaga Mushroom Extract}

In order to evaluate the immunological activity of the CMW and CME, we tested the nuclear factor of the activated T cell (NFAT) assay. The CTLA-4 Jurkat T cells have a NFAT response when CTLA-4/CD80 is blocked by anti-CTLA-4 antibodies or small molecules sent to signal expression of luciferase. Once luciferase is expressed, it produces light in an enzymatic assay, and the amount of light can be measured. CMW and CME were treated to a four-fold dilution $(1.56 \mu \mathrm{g} / \mathrm{mL}$ to $12.5 \mu \mathrm{g} / \mathrm{mL})$ on a co-culture well plate with CTLA-4 Jurkat T cells: CD80/aAPC CHO-K1 cells (1:1). We observed that the CMW and CME blocked CTLA-4/CD80 interaction. The luminescence of the CTLA-4/CD80 reporter assays that were treated with CMW and CME significantly increased ( $\sim 3$ fold and $\sim 4.5$ fold, respectively) at $12.5 \mu \mathrm{g} / \mathrm{mL}$, higher than the negative control "cell only", with $\mathrm{EC}_{50}$ values of $3.12 \mu \mathrm{g} / \mathrm{mL}$ and $3.95 \mu \mathrm{g} / \mathrm{mL}$, respectively. These results supported our observation of the ELISA assay results (Figure 2). 
A

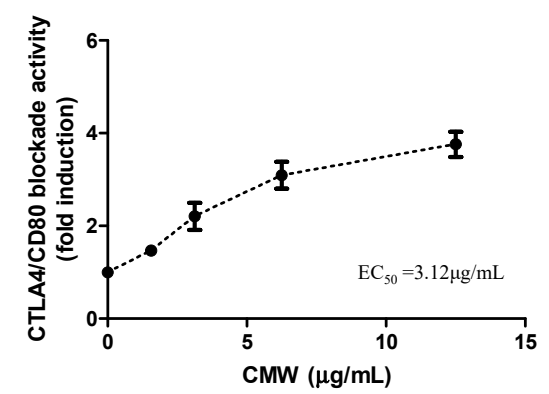

B

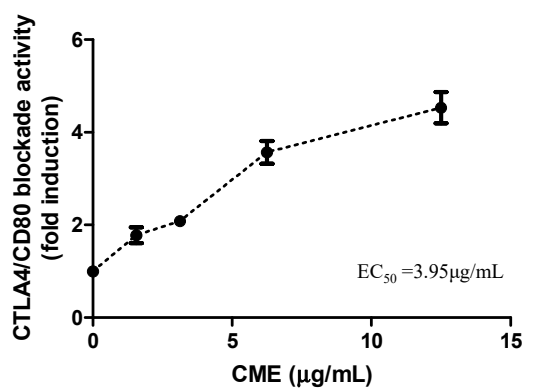

Figure 2. Nuclear factor of the activated T cell (NFAT) luciferase reporter activity by CTLA-4/NFAT Reporter-Jurkat $\mathrm{T}$ cells treated with Chaga mushroom/water extract (CMW) and Chaga mushroom/70\% EtOH extract (CME). Fold luminescence of Jurkat T cells treated with CMW (A) and CME (B) at several concentrations (1.56 to $12.5 \mu \mathrm{g} / \mathrm{mL}$ ). The half-maximal effective concentration $\left(\mathrm{EC}_{50}\right)$ was calculated using the Prism "log[agonist] vs. normalized response" equation. All results are presented as the mean of three independent biological replicates and compared with the vehicle control group.

\subsection{Evaluation of Cytotoxicity of the Chaga Mushroom Extract in CTLA-4 Jurkat T and CD80/aAPC} CHO-K1 Cells

To identify how CMW and CME can affect the cell viability of both CTLA-4 Jurkat T cells and CD80/aAPC CHO-K1 cells, they were used in the cell-based assay for CTLA-4/CD80 inhibitor screening. Several concentrations of CMW and CME were treated on CTLA-4 Jurkat T and CD80/aAPC CHO-K1 cells for $24 \mathrm{~h}$. Then, the cell viability was assessed using a Cell Counting Kit-8 (CCK) assay. In all tested concentrations, neither the CMW nor the CME showed an effect on the cell viability of CTLA-4 Jurkat $\mathrm{T}$ cells or CD80/aAPC CHO-K1 cells. These results showed no cytotoxicity at the concentrations used in the previously described NFAT reporter assay (Figure 3).

A
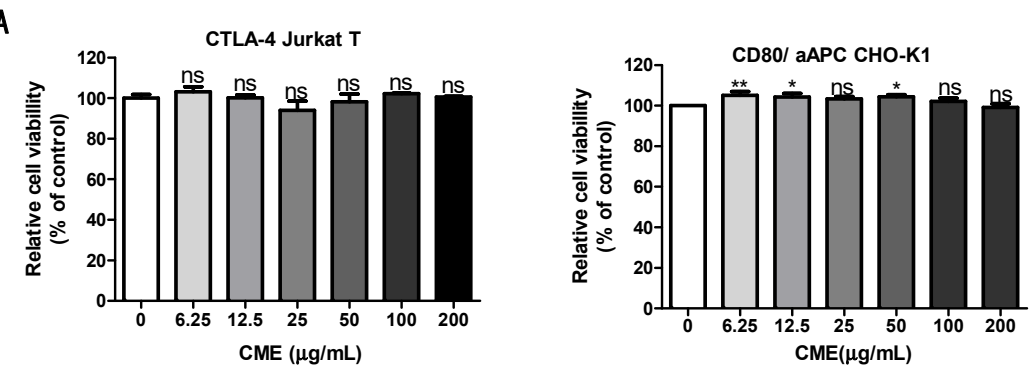

B
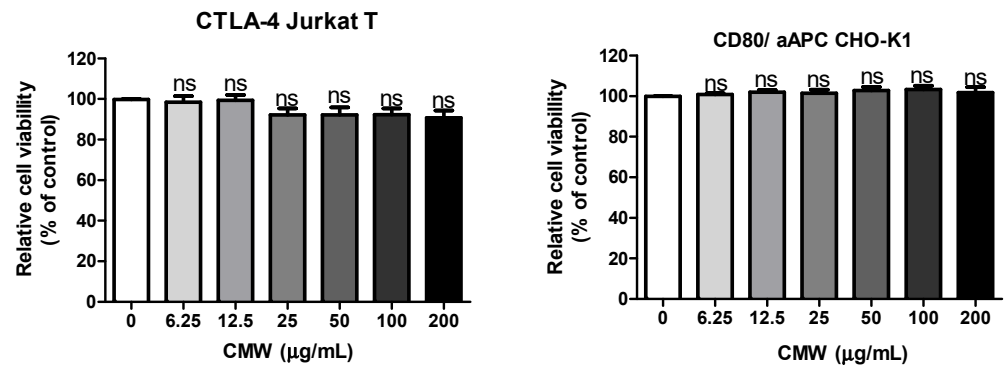

Figure 3. Cell viability effects of the Chaga mushroom/water extract (CMW) and Chaga mushroom/70\% EtOH extract (CME) on CTLA-4 Jurkat T and CD80/aAPC CHO-K1 cells. CME (A) and CMW (B) were treated with various concentrations $(6.25$ to $200 \mu \mathrm{g} / \mathrm{mL})$ on CTLA-4 Jurkat T and CD80/aAPC CHO-K1 cells for $24 \mathrm{~h}$, and the cell viability was assessed using a CCK 8 assay. All results are presented as the mean of three independent biological replicates, with ${ }^{*} p<0.05,{ }^{* *} p<0.01$, compared with the vehicle control group. 


\subsection{Content Analysis of Lanosterol (1)}

We investigated the amount of lanosterol (1) in the 70\% EtOH extract of the Chaga mushroom that showed blocking of the CTLA-4/CD80 interaction via HPLC-CAD analysis. The calibration curve of the lanosterol (1) was $y=0.2003 x+3.9707$ with good linearity $\left(r^{2}=0.9981\right)$ in the concentration range tested $(25-200 \mu \mathrm{g} / \mathrm{mL})$. The LODs and LOQs of the lanosterol (1) were 0.1192 and $0.0477 \mu \mathrm{g} / \mathrm{mL}$, respectively. To confirm the lanosterol in the sample chromatogram, we compared the results with the standard solution retention time, $29.14 \mathrm{~min}$, and the UV spectrum (Figure 4). The content of lanosterol (1) in the $70 \% \mathrm{EtOH}$ extract of Chaga mushroom was $0.41 \%$ (Figure 4). Consistent with the results of Chaga mushroom extract (Figure 1), lanosterol also interfered the interaction of CTLA-4 and CD80 in a dose-dependent manner (Figure S1). Considering this result, lanosterol might be suggested as one of active compound that blocked CTLA4/CD80 interaction in CME.

A

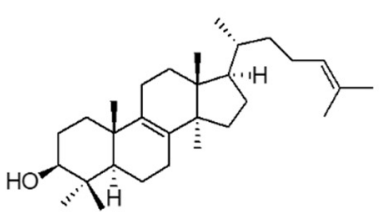

B

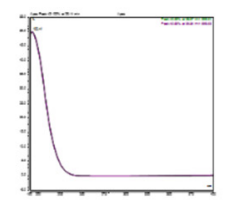

STD

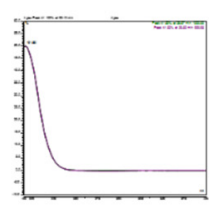

CME

C

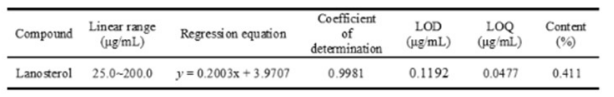

D
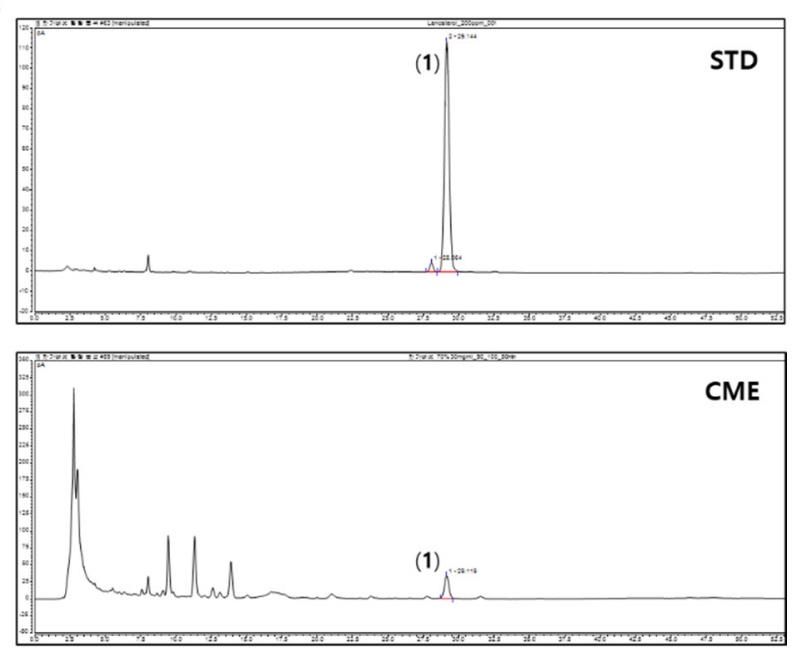

Figure 4. Structure of lanosterol (1) (A); UV spectra of the standard compound of lanosterol (1) and $70 \%$ ethanol extract (B); regression equations, LODs, and LOQs of lanosterol (C); and HPLC-CAD chromatograms (D).

\section{Discussion}

In this study, we performed an ELISA-binding assay of CME and CMW on the blocking of the CTLA-4/CD80 interaction in a concentration-dependent manner. Additionally, to evaluate whether the CME and CMW can promote T cell functional activity using the CTLA-4/CD80 blockade bioassay, both extracts were used to enhance $\mathrm{T}$ cell activation and increase the expression of luciferase. Moreover, we confirmed the constituents of the Chaga mushroom/70\% ethanol extract via HPLC-CAD analysis.

Chaga mushroom (I. obliquus) has been used as a traditional therapy in Asia for a long time [1]. The anticancer effects of I. obliquus extract on various types of cells, including melanoma cells, human lung carcinomas, and human liver cancer cells, have been researched and reported [15-17]. However, the blocking effect of the immune checkpoint against Chaga mushroom extract is not understood.

The CTLA-4/CD80 inhibitory effect and T cell activation effects of CME and CMW extracts were confirmed using several cell-based assays. Competitive ELISA results showed that CME and CMW blocked CTLA-4/CD80 interaction in a concentration-dependent manner. It was also confirmed through CTLA-4 blocking assays that CMW and CME interfere with CTLA-4/CD80 interaction and activate T cells. CMW and CME were concentration-dependent, and did not show cell cytotoxicity in CTLA-4 Jurkat T and CD80/aAPC CHO-K1 cells. We conducted HPLC-CAD analysis to confirm the content of lanosterol. According to the standard curve solution, the amount of lanosterol (1) was diluted with methanol at each concentration. The established HPLC-CAD method was suitable for analysis of lanosterol (Figure 4). Lanosterol is present in the Chaga mushroom/ethanol extract at $0.41 \%$. Lanosterol is one of the tetracyclic triterpenoids, and is the precursor to all animal and fungal steroids. Steroids 
are hydrophobic compounds that have very low solubility in water, and we showed that lanosterol was detected only in the ethanol extract but not the water extract. As shown in Supplementary Material Figure S1, lanosterol tested competitive ELISA and it also blocked the interaction of CTLA-4/CD80. Although lanosterol has been suggested as one of active compound for Chaga mushroom ethanol extract, however, we will investigate other immune checkpoint inhibitors in the CMW extract in a future study.

Chaga mushrooms stimulate the immune system and show antitumor activity against diverse types of cancer [15-20]. In addition, the Chaga mushroom blocks CTLA-4/CD80 interaction and stimulates $\mathrm{T}$ cell activity. Terpenoids isolated from the herb medicinal plant showed immunological activity. [21] Triterpenoids isolated from the Chaga mushroom have been reported to show a variety of bioactivities, including those mentioned in ref. [22,23]. Thus, we suggest that the blocking effect of CME on the CTLA-4/CD80 interaction can be attributed to the abundant triterpenoid constituents in the CME. According to a previous study [24], adverse effects, such as oxalate nephropathy, can occur after the long-term usage of Chaga mushrooms. Administration of Chaga mushroom should be carefully monitored in patients with kidney function disorders, due to contraindications and side effects. In addition, Chaga mushrooms may interact with drugs such as aspirin and clopidogel, given that the Chaga mushroom inhibits platelet aggregation [25].

\section{Conclusions}

We have investigated the immune check point inhibitor of herbal medicines. The blocking of the immune check point CTLA-4/CD80 interaction by the Chaga mushroom was confirmed using several cell experiments and HPLC analysis. The Chaga mushroom extracts did not show cell cytotoxicity, blocking of the CTLA-4/CD80 interaction, or activation of T cells. Our findings suggest that the Chaga mushroom can be developed as a new immune check point inhibitor. In future research, we will conduct an in vivo study to test the inhibitory effect of another triterpenoid compound.

Supplementary Materials: The following are available online at http://www.mdpi.com/2076-3417/10/17/5774/s1, Figure S1: Blocking effect of CTLA-4/CD80 interaction of lanosterol.

Author Contributions: Conceptualization and revised the paper: H.-S.C.; Performed experiments: T.I.K., J.H.K. and J.-G.C.; Analyzed the data: T.I.K. and W.L.; Wrote the paper: T.I.K. All authors have read and agreed to the published version of the manuscript.

Funding: This research was supported by Grant number KSN2013230 from the KIOM (Korea Institute of Oriental Medicine), provided by the Ministry of Science and ICT, Republic of Korea. The present study was supported by Basic Science Research Program through the National Research Foundation (NRF) funded by the Ministry of Education (Grant number NRF2020R1C1C1006749), Republic of Korea.

Conflicts of Interest: The authors declare no conflict of interest.

\section{References}

1. Ma, L.; Chen, H.; Dong, P.; Lu, X. Anti-inflammatory and anticancer activities of extracts and compounds from the mushroom Inonotus obliquus. Food Chem. 2013, 139, 503-508. [CrossRef] [PubMed]

2. Park, Y.M.; Won, J.H.; Kim, Y.H.; Choi, J.W.; Park, H.J.; Lee, K.T. In vivo and in vitro anti-inflammatory and anti-nociceptive effects of the methanol extract of Inonotus obliquus. J. Ethnopharmacol. 2005, 101, 120-128. [CrossRef] [PubMed]

3. Lee, H.S.; Kim, E.J.; Kim, S.H. Ethanol extract of Innotus obliquus (Chaga mushroom) induces G1 cell cycle arrest in HT-29 human colon cancer cells. Nutr Res. Pract. 2015, 9, 111-116. [CrossRef] [PubMed]

4. Baek, J.; Roh, H.S.; Baek, K.H.; Lee, S.; Lee, S.; Song, S.S.; Kim, K.H. Bioactivity-based analysis and chemical characterization of cytotoxic constituents from Chaga mushroom (Inonotus obliquus) that induce apoptosis in human lung adenocarcinoma cells. J. Ethnopharmacol. 2018, 224, 63-75. [CrossRef]

5. He, J.; Feng, X.Z.; Lu, Y.; Zhao, B. Three new triterpenoids from Fuscoporia obliqua. J. Asian Natl. Prod. Res. 2001, 3, 55-61. [CrossRef] 
6. Glamoclija, J.; Ciric, A.; Nikolic, M.; Fernandes, A.; Barros, L.; Calhelha, R.C.; Ferreira, I.C.; Sokovic, M.; van Griensven, L.J. Chemical characterization and biological activity of Chaga (Inonotus obliquus), a medicinal "mushroom". J. Ethnopharmacol. 2015, 162, 323-332. [CrossRef]

7. Song, Y.; Hui, J.; Kou, W.; Xin, R.; Jia, F.; Wang, N.; Hu, F.; Zhang, H.; Liu, H. Identification of Inonotus obliquus and analysis of antioxidation and antitumor activities of polysaccharides. Curr. Microbiol. 2008, 57, 454-462. [CrossRef]

8. Kim, Y.J.; Park, J.; Min, B.S.; Shim, S.H. Chemical Constituents from the Sclerotia of Inonotus obliquus. J. Korean Soc. Appl. Biol. Chem. 2011, 54, 287-294. [CrossRef]

9. Li, W.; Kim, T.I.; Kim, J.H.; Chung, H.S. Immune Checkpoint PD-1/PD-L1 CTLA-4/CD80 are Blocked by Rhus verniciflua Stokes and its Active Compounds. Molecules 2019, 24, 4062. [CrossRef]

10. June, C.H.; Warshauer, J.T.; Bluestone, J.A. Is autoimmunity the Achilles' heel of cancer immunotherapy? Nat. Med. 2017, 23, 540-547. [CrossRef]

11. Sasikumar, P.G.; Ramachandra, M. Small-Molecule Immune Checkpoint Inhibitors Targeting PD-1/PD-L1 and Other Emerging Checkpoint Pathways. BioDrugs 2018, 32, 481-497. [CrossRef] [PubMed]

12. Kourie, H.R.; Klastersky, J. Immune checkpoint inhibitors side effects and management. Immunotherapy 2016, 8, 799-807. [CrossRef] [PubMed]

13. Linsley, P.S.; Brady, W.; Urnes, M.; Grosmaire, L.S.; Damle, N.K.; Ledbetter, J.A. CTLA-4 is a second receptor for the B cell activation antigen B7. J. Exp. Med. 1991, 174, 561-569. [CrossRef]

14. Hurwitz, A.A.; Foster, B.A.; Kwon, E.D.; Truong, T.; Choi, E.M.; Greenberg, N.M.; Burg, M.B.; Allison, J.P. Combination immunotherapy of primary prostate cancer in a transgenic mouse model using CTLA-4 blockade. Cancer Res. 2000, 60, 2444-2448. [PubMed]

15. Youn, M.J.; Kim, J.K.; Park, S.Y.; Kim, Y.; Park, C.; Kim, E.S.; Park, K.I.; So, H.S.; Park, R. Potential anticancer properties of the water extract of Inonotus obliquus by induction of apoptosis in melanoma B16-F10 cells. J. Ethnopharmacol. 2009, 121, 221-228. [CrossRef]

16. Lemieszek, M.K.; Langner, E.; Kaczor, J.; Kandefer-Szerszen, M.; Sanecka, B.; Mazurkiewicz, W.; Rzeski, W. Anticancer effects of fraction isolated from fruiting bodies of Chaga medicinal mushroom, Inonotus obliquus (Pers.:Fr.) Pilat (Aphyllophoromycetideae): In vitro studies. Int J. Med. Mushrooms 2011, 13, 131-143. [CrossRef]

17. Youn, M.J.; Kim, J.K.; Park, S.Y.; Kim, Y.; Kim, S.J.; Lee, J.S.; Chai, K.Y.; Kim, H.J.; Cui, M.X.; So, H.S.; et al. Chaga mushroom (Inonotus obliquus) induces G0/G1 arrest and apoptosis in human hepatoma HepG2 cells. World J. Gastroenterol. 2008, 14, 511-517. [CrossRef]

18. Nguyet, T.M.N.; Lomunova, M.; Le, B.V.; Lee, J.S.; Park, S.K.; Kang, J.S.; Kim, Y.H.; Hwang, I. The mast cell stabilizing activity of Chaga mushroom critical for its therapeutic effect on food allergy is derived from inotodiol. Int. Immunopharmacol. 2018, 54, 286-295. [CrossRef]

19. Niu, H.; Song, D.; Mu, H.; Zhang, W.; Sun, F.; Duan, J. Investigation of three lignin complexes with antioxidant and immunological capacities from Inonotus obliquus. Int. J. Biol. Macromol. 2016, 86, 587-593. [CrossRef]

20. Won, D.P.; Lee, J.S.; Kwon, D.S.; Lee, K.E.; Shin, W.C.; Hong, E.K. Immunostimulating activity by polysaccharides isolated from fruiting body of Inonotus obliquus. Mol. Cells 2011, 31, 165-173. [CrossRef]

21. Rios, J. Effects of triterpenes on the immune system. J. Ethnopharmacol. 2010, 128, 1-14. [CrossRef] [PubMed]

22. Zhao, F.; Mai, Q.; Ma, J.; Xu, M.; Wang, X.; Cui, T.; Qiu, F.; Han, G. Triterpenoids from Inonotus obliquus and their antitumor activities. Fitoterapia 2015, 101, 34-40. [CrossRef] [PubMed]

23. Nikitina, S.A.; Habibrakhmanova, V.R.; Sysoeva, M.A. Chemical Composition and Biological Activity of Triterpenes and Steroids of Chaga Mushroom. Biomed. Khim 2016, 10, 63-69. [CrossRef]

24. Kikuchi, Y.; Seta, K.; Ogawa, Y.; Takayama, T.; Nagata, M.; Taguchi, T.; Yahata, K. Chaga mushroom-induced oxalate nephropathy. Clin. Nephrol. 2014, 81, 440-444. [CrossRef] [PubMed]

25. Hyun, K.W.; Jeong, S.C.; Lee, D.H.; Park, J.S.; Lee, J.S. Isolation and characterization of a novel platelet aggregation inhibitory peptide from the medicinal mushroom, Inonotus obliquus. Peptides 2006, 27, 1173-1178. [CrossRef]

(C) 2020 by the authors. Licensee MDPI, Basel, Switzerland. This article is an open access article distributed under the terms and conditions of the Creative Commons Attribution (CC BY) license (http://creativecommons.org/licenses/by/4.0/). 